\title{
In response to the article entitled "Surgery for recurrent lower lip carcinoma" by Maharjan S et al, published in KUMJ, 2008 Vol. 6, No. 3, Issue 23, 375-378.
}

Bhandary S

Department of ORL and HNS, BPKIHS, Dharan

$\mathrm{I}$ would like to add few salient things and recent updates regarding management of oral cancer including verrucous carcinoma based on evidence based practice which in turn would raise several queries regarding overall approach and management of this 34 year young adult presenting with verrucous cancer of lower lip, who subsequently had several local and regional recurrences.

Verrucous carcinoma (VC) first described in 1948 by Lauren V. Ackerman is a distinct variant of differentiated squamous cell carcinoma (SCC) with low grade malignancy, slow growth and no or only low metastatic potential. ${ }^{1}$ It is often associated with long-term use of smokeless tobacco although examples occur among nonusers as well. Betel nut chewing, poor dental hygiene and Human Papilloma Virus (HPV) infection have been implicated in the development of oral VC. Various premalignant conditions like SMF, leukoplakia, lichen planus etc have been associated with this condition ${ }^{1}$.

Difficulties remain as to the appropriate classification of those lesions with dominant features of $\mathrm{VC}$ which also contain small foci of squamous cell carcinoma. In $20 \%$ of VC coexistent foci of less-differentiated SCC could be found. No matter what the treatment is, the rate of local recurrences is said to be high ranging from $30 \%$ to $50 \%$ and not unusually is the result of inadequate surgery but because of the size of the tumor and left dysplasia close to the verrucous carcinoma. Recently successfull treatment of an extensive VC with intra-arterial infusion of methotrexate or topical 5-aminolevulinic acid-mediated photodynamic therapy was reported ${ }^{1}$.

There have been evidences in literatures which clearly states that surgery is the treatment of choice for all oral cavity tumors (except early lip cancers) and radiotherapy (RT) is reserved only for advanced stage III or IV diseases as adjuvant therapy or in cases where the histopathology reveals close or positive margins, high grade tumor, thick tumor infiltrating muscles and soft tissues or perineural extension. RT is also justified in large bulky nodes, multiple level nodal involvement and metastatic node showing perinodal extension. This modality of treatment is usually avoided in young patients in view of the risk of radiation induced cancer unless the lesion is inaccessible or the surgery is mutilating, like in cases of oropharyngeal and nasopharyngeal carcinomas. ${ }^{2}$

In the article the author has not very clearly mentioned if the patient was managed at the same institution or outside in the past. The fact that there was various subsites of oral cavity involved subsequently should also raise possibility of field cancerization in this patient. The clinical details including the size, proper site in lower lip and staging of disease, rational for various treatment modalities this patient was being subjected to and also histopathological reports (HPR) are missing in the case report.

The author has mentioned that margins could not be commented upon due to lack of frozen section facility, whereas it could have been looked for in HPR subsequently, a pathological staging of disease made and managed accordingly. The patient here has been subjected to radiotherapy without any rationale as to the stage of disease or the margins of specimen, whereas the author himself has stated that HPR revealed no invasion of muscles or lymphovascular invasion.

Another important aspect of management of any head and neck malignancy is that the treatment of the neck should not be seen in isolation but rather in the context of the overall management plan $^{2}$. The appropriate management of the clinically negative neck among patients with SCC of the oral cavity and oropharynx continues to be a therapeutic challenge.

In the oral cavity and oropharynx, the incidence of occult nodal disease varies broadly with the tumor site and stage. More than $50 \%$ of patients with squamous cell carcinoma of the oral cavity have lymph node 
metastases and histological confirmation of metastatic disease is the most important prognostic factor. ${ }^{3}$ The high incidence rates of occult cervical metastases (> $20 \%-25 \%$ in $\mathrm{T} 2$ disease) in oral cavity cancers have been reported in literature. ${ }^{3}$ Among patients with a clinically negative neck, the incidence of occult metastases varies with the site, size and thickness of the primary tumour. Additional factors that may predict possible occult nodal involvement in oral cancers are tumor grade, vascular invasion, depth of invasion, and DNA aneuploidy. It has been recommended that an appropriate threshold for treatment of the clinically negative neck is when there is a $15 \%$ - $20 \%$ or greater risk of occult disease. ${ }^{2,4}$ This recommendation was based on an analysis of the utility of the management options taking into account the incidence of node involvement, complications of treatment, and disease control rates. This threshold would include most oral cavity cancers staged T2 or higher and nearly all oropharyngeal cancers, including those staged $\mathrm{T} 1$.

In support of elective neck treatment is the fact that when patients initially managed by observation subsequently return with nodal involvement, their metastatic disease is often advanced, making regional control more difficult. In addition, the increasing use of microvascular free flaps for reconstruction in the oral cavity and oropharynx mean that the neck is very frequently entered for the reconstructive procedure, and therefore it is appropriate to remove the at-risk nodes during supraomohyoid neck dissection (SND). Several retrospective studies and one randomized clinical trial have shown SND of levels I through III to be highly efficacious and widely accepted as the appropriate selective neck dissection for patients with oral cavity cancer and a clinically negative neck. There has been however a recent debate about whether level IV should be included or not. The risk of contralateral occult neck involvement in the oral cavity squamous cell carcinomas above the T3 stage or those crossing the midline with unilateral metastases was high, and patients who presented with a contralateral metastatic neck had a worse prognosis than those whose disease was staged as N0. Therefore, in literatures they advocate an elective contralateral neck treatment with surgery or radiotherapy in patients with oral cavity squamous cell carcinoma with ipsilateral node metastases or tumors, or both, whose disease is greater than $\mathrm{T} 3$ or crossing the midline. ${ }^{4,5}$

The evidence based guideline for managing cancer includes proper diagnosis and staging, appropriate treatment modality after discussing with the patient regarding options, risk, benefits and oncological safety while performing surgery which includes taking adequate margins and performing a good reconstruction. Functional restoration and quality of life should be taken into consideration as far as practicable while treating the patient. At no point should the oncological safety be compromised while managing the disease. HPR details and radiological evaluation are invaluable in decision making as well as overall management of the case.

Gillies, Webstes'r flap and other reconstructive methods have been well accepted and described in the past literature for reconstruction of lip and oral cavity defects post-operatively. In the recent years free flaps have been a major advance in oral cavity defect reconstruction, where well trained plastic surgery team is available. However the basic principle of planning any flaps would be to ensure that resection has been complete with adequate mucosal as well as bony and soft tissue cut margins and taking care of regional metastasis.

The pictures of recurrence shown of this patient clearly reveal disease extending from one angle of mouth to the other as well as presence of paramandubular disease. The need for marginal mandibulectomy here further upstages the disease to $\mathrm{T}_{3}$ or $\mathrm{T}_{4}$ where probability of nodal metastasis is as high as $40-60 \%$. Moreover this was a recurrent disease which is known for aggressive behavior, hence requires equally aggressive approach. The ideal in above situation would have been to perform bilateral supraomohyoid neck dissection (SOHD), frozen section if possible and then proceed further for modified neck dissection (MND) if any nodes come positive. Where frozen section facilities are not available one can explain to the patient preoperatively about the risk of nodal metastasis, seek consent and go for an upfront MND. Finally, a policy of observation can be used selectively and applied mainly to patients with early stage disease who require close follow-up. However, a likelihood of poor follow-up is believed to be a valid reason for electively treating the neck. ${ }^{2}$

In this young man the neck should definitely have been addressed at the time of surgery and before contemplating any kind of reconstruction. We have seen that patient has come back with nodal disease within 3 months. Any recurrence less than 6 months interval has graver prognosis. ${ }^{4}$ The author has also failed to mention what has the duration of follow up had been in this case after last surgery as any cancer requires close follow up for at least 5 years and most recurrences occur within a period of 6 months to 2 years.

It is a well accepted fact that any article published should have some take home message for readers and contribute to the literature in some way. The intentions of the comments are not to challenge anyone's skill and abilities here. However, considering that the disease was recurrent (for the fourth time) in this young patient, lack of proper clinical, histopathological and surgical details and approach, adopting a well established reconstruction 
method without considering the complete oncological clearance does make one critically view the objective and the message the author wishes to highlight through this article.

\section{References}

1. Walvekar R R, Chaukar DA, Deshpande MS, Pa PS, Chaturvedi P, Kakade A, Kane SV and D'Cruz AK Verrucous carcinoma of the oral cavity: A clinical and pathological study of 101 cases Oral Oncol. 2009 Jan;45(1):47-51.

2. O'Brien CJ, Traynor SJ, McNeil E, McMahon JD, Chaplin JM. The use of clinical criteria in the management of the clinically negative neck among patients with squamous cell carcinima of the oral cavity and oropharynx Archives of Otolaryngol Head and Neck Surg. 2000;126(3) 360-5
3. Kowalski LP, Sanabria A. Elective neck dissection in oral carcinoma: a critical review of the evidence Acta Otorhinolaryngol Ital. 2007 ; (3):113-7

4. Scwartz GJ, Mehta RH, Weinig BL, Shaligram C, Portugal LG. Salvage treatment for recurrent scuamous cell carcinoma of the oral cavity. Head and Neck 2000;22(1):34-41

5. Koo BS, Lim YC, Lee JS, Choi EC. Management of contralateral N0 neck in oral cavity squamous cell carcinoma Head and Neck2006; 28(10):896-901 